\title{
Pediatric patient with Sleep Disordered Breathing (SDB) of central origin secondary to Arnold Chiari type I malformation: a case report.
}

\author{
Diego Morena Valles ${ }^{1}$, Sofia Romero Peralta ${ }^{1}$, and Olga Mediano ${ }^{1}$ \\ ${ }^{1}$ Hospital General Universitario de Guadalajara
}

June 25, 2020

\begin{abstract}
The diagnosis and treatment of Sleep Disordered Breathing (SDB) in pediatric age differs in relation to the adult. Central sleep apnea syndrome (CSAS) is less frequent in childhood, and one of the causes of it is the alterations of the brainstem like the malformation of Chiari type I. The main diagnostic method of SDB is Polysomnography, but there are other methods like the brain magnetic resonance imaging (MRI). Here, we share our experience with an 8-year-old female with malformation of Chiari type I and CSAS, and the steps we follow for the diagnosis of such an infrequent pathology in childhood.
\end{abstract}

\section{Introduction}

The prevalence of Obstructive Sleep Apnea (OSA) in children under 5 years is around $3 \%$, with a peak incidence between 2 and 5 years $^{1}$, being much less frequent in the case of central sleep apnea syndrome (CSAS).

Combinations of anatomical and functional factors influence the pathogenesis of childhood OSA. Among the causes of CSAS, diseases of the central nervous system, alterations of the brainstem (tumors, malformation of Chiari type I and type II, stenosis of the foramen magno) and the use of sedative or narcotic medication stand out ${ }^{1,3}$.

The current diagnostic reference method for any SDB in children is Polysomnography (PSG), although Cardiorespiratory Polygraphy (CRP) could also be performed if necessary as a first diagnostic approach if the symptomatology is highly suggestive. Other diagnostic methods are also useful for knowing the etiology of SDB such as brain MRI ${ }^{4}$.

The treatment of OSA in children is mainly based on the etiology of the disease. In the case of CSAS, the treatment of choice is that of the underlying pathology, so its identification is essential ${ }^{3,4,5}$.

Here, we describe a childhood patient with a diagnosis of moderate mixed OSA of central predominance, whose probable etiology, Arnold Chiari Type I syndrome, was discovered by performing a brain MRI. This case provides a bibliography on how to act and what steps to follow when diagnosing a CSAS in childhood.

\section{Case}

We report the case of an 8-year-old woman, native from Spain, with no personal history of interest. She has a family history of a mother with a diagnosis of severe OSA in treatment with nocturnal CPAP, a 14 years old sister with a diagnosis of severe OSA and in treatment with nocturnal CPAP and 11 years old brother with a diagnosis of moderate OSA. 
The patient went to the Guadalajara University Hospital's Sleep Unit consultations of the Pneumology Service for restless sleep symptoms, apnea witnessed accompanied by persistent snoring, headache and bruxism. He did not refer to nocturnal enuresis, and presented a good school performance. Physical examination highlights tonsillar hypertrophy III / IV with normal palate and cardiopulmonary auscultation without alterations. In December 2017, with 6 years, hospital CRP was performed with a result of severe OSA of obstructive predominance (hypopnea apneas index-AHI 16 / h: obstructive AHI: 15.3 ; central AHI: 0.7 / h). Tonsillectomy was decided, after which the patient presented clinical improvement with decreased daytime hypersomnolence.

Postamigdalectomy CRP was performed in October 2018 with a diagnosis of moderate mixed OSA of central predominance (AHI: 9.3 / h: obstructive AHI: 3.3 / h; central AHI $6 /$ h). We request a new evaluation by Otolaryngology, with endoscopy with small non-obstructive adenoid remains. Given the results of postmigralectomy CRP, it was decided to perform hospital PSG presenting a change in the sleep architecture for the patient's age, increased surface sleep and frequent daydreams, very frequent respiratory events were recorded accompanied by desaturations of relevance and long duration (with no hypoventilation observed) . These events are about sleep apnea with a clear central predominance (AHI: 26.7 / h, obstructive AHI: 8.4 / h, central AHI: 18.3 / h) (Image 1).

Given this diagnosis, an etiological study of the CSAS was initiated, performing cerebral-cervical-dorsal MRI with contrast. In that MRI, a $9 \mathrm{~mm}$ descent of the cerebellar tonsils was observed below the plane of the great hole compatible with Arnold Chiari type I malformation, with association to cervical syringomyelia that extended from C5 to D9 (Image 2). The patient was referred to the neurosurgery department for evaluation of surgical treatment.

\section{Discussion}

SDB in pediatric age differ both in the definition, diagnosis and treatment in relation to the adult. The child presents anatomical and functional peculiarities in the airway along with maturational characteristics from the point of view of sleep neurophysiology ${ }^{2}$.

Tonsillar hypertrophy is the main nasal and oropharyngeal anatomical pathogenesis' factors of childhood OSA. Also included in the different factors that cause this pathogenesis are less frequent ones such as obesity, dental malocclusion or craniofacial malformations ${ }^{2}$.

The clinical manifestations of OSA are very varied depending on the severity, etiologies and the different degree of development, but the most frequent are snoring, evidence of nocturnal apnea, daytime hyperactivity or hypersomnolence, poor school performance and neurocognitive alterations ${ }^{1}$. In the case of CSAS, the symptoms are less florid and usually occur asymptomatically.

Although PSG and CRP are the diagnostic techniques of choice, it is important to know that other methods are also interesting. A brain MRI may be an important diagnostic tool for unexplained sleep disorders in nonsyndromic and syndromic children, and it should be considered in the context of an unexplained SDB, including OSA in the absence of adenotonsillar hypertrophy, persistent OSA following adenotonsillar, central sleep apnea, and nocturnal hypoventilation syndrome ${ }^{4}$.

In our case, the realization of a brain MRI showed a probable origin of the pathology. The malformation of Chiari type I is characterized by the existence of a decrease in the cerebellar tonsils that are located below the foramen magnum, associated with compressive phenomena of the brainstem and upper spinal cord. Ferré et al ${ }^{2}$ described that the most frequent clinical manifestations are usually occipitonucal headaches, dizziness and nocturnal respiratory disturbances, such as central apneas.

Adeno-tonsillectomy is the most frequent technique of treatment in children's OSA, with an improvement in AHI of $75 \%^{1}$. Other non-surgical treatments would be the use of CPAP, weight loss, maxillary expansion and / or orthodontic and other methods aimed at eliminating the possible etiological trigger ${ }^{1}$. In the case of CSAS, it is important to identificate the underlying pathology. In Arnold Chiari malformation, it is usually decompressive neurosurgery ${ }^{5}$. 
In summary, the Arnold Chiari malformation is a rare cause of central apneas in the pediatric patient, which requires an MRI study for diagnosis. It is important to assess these possible causes in a case of pediatric SAC. Also we think that it would be useful to carry out studies in Chiari malformation's patients to evaluate respiratory alterations before and after surgery to determine whether surgery treats SDB.

Image 1 : PSG where multiple apneas of central origin are evident.

Image 2 : MRI of T2 sagittal brain showing a $9 \mathrm{~mm}$ decrease in cerebellar tonsils below the plane of the great hole compatible with Arnold Chiari type I malformation (red arrow), with association to cervical syringomyelia that extends from C5 until D9 (yellow arrow).

\section{Bibliography}

1. Leu RM. Sleep-Related Breathing Disorders and the Chiari 1 Malformation. Chest. 2015 Nov;148(5):13461352. doi: 10.1378/chest.14-3090.

2. Ferré Á, Poca MA, de la Calzada MD, Moncho D, Romero O, Sampol G, Sahuquillo. J. Sleep-Related Breathing Disorders in Chiari Malformation Type 1: A Prospective. Study of 90 Patients. Sleep. 2017 Jun 1;40(6). doi: 10.1093/sleep/zsx069. PubMed. PMID: 28453783.

3. Zaffanello M, Sala F, Sacchetto L, Gasperi E, Piacentini G. Evaluation of the central sleep apnea in asymptomatic children with Chiari 1 malformation: an open question. Childs Nerv Syst. 2017 May;33(5):829832. doi: 10.1007/s00381-017-3399-9. Epub 2017 Apr 5. PubMed PMID: 28382434.

4. Selvadurai S, Al-Saleh S, Amin R, Zweerink A, Drake J, Propst EJ, Narang I. Utility of brain MRI in children with sleep-disordered breathing. Laryngoscope. 2017 Feb;127(2):513-519. doi: 10.1002/lary.26042. Epub 2016 May 2. PubMed PMID: 27140660.

5. Botelho RV, Bittencourt LR, Rotta JM, Tufik S. The effects of posterior fossa decompressive surgery in adult patients with Chiari malformation and sleep apnea. J Neurosurg. 2010 Apr;112(4):800-7. doi: 10.3171/2009.7.JNS09174. PubMed PMID: 19663555.

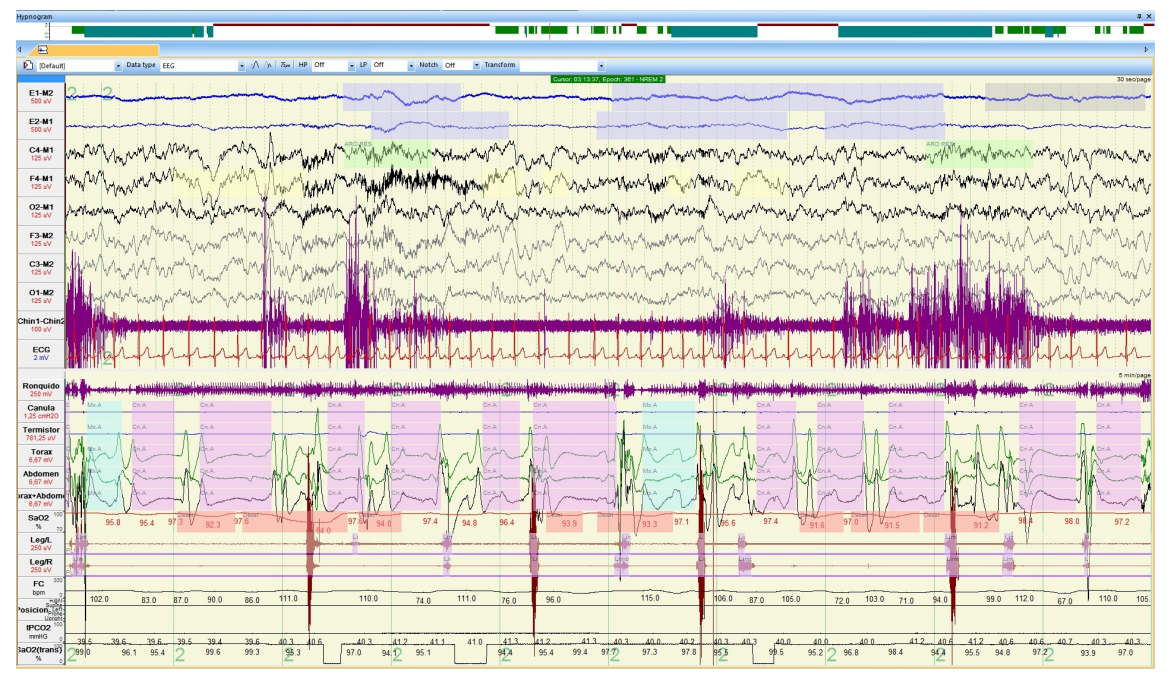




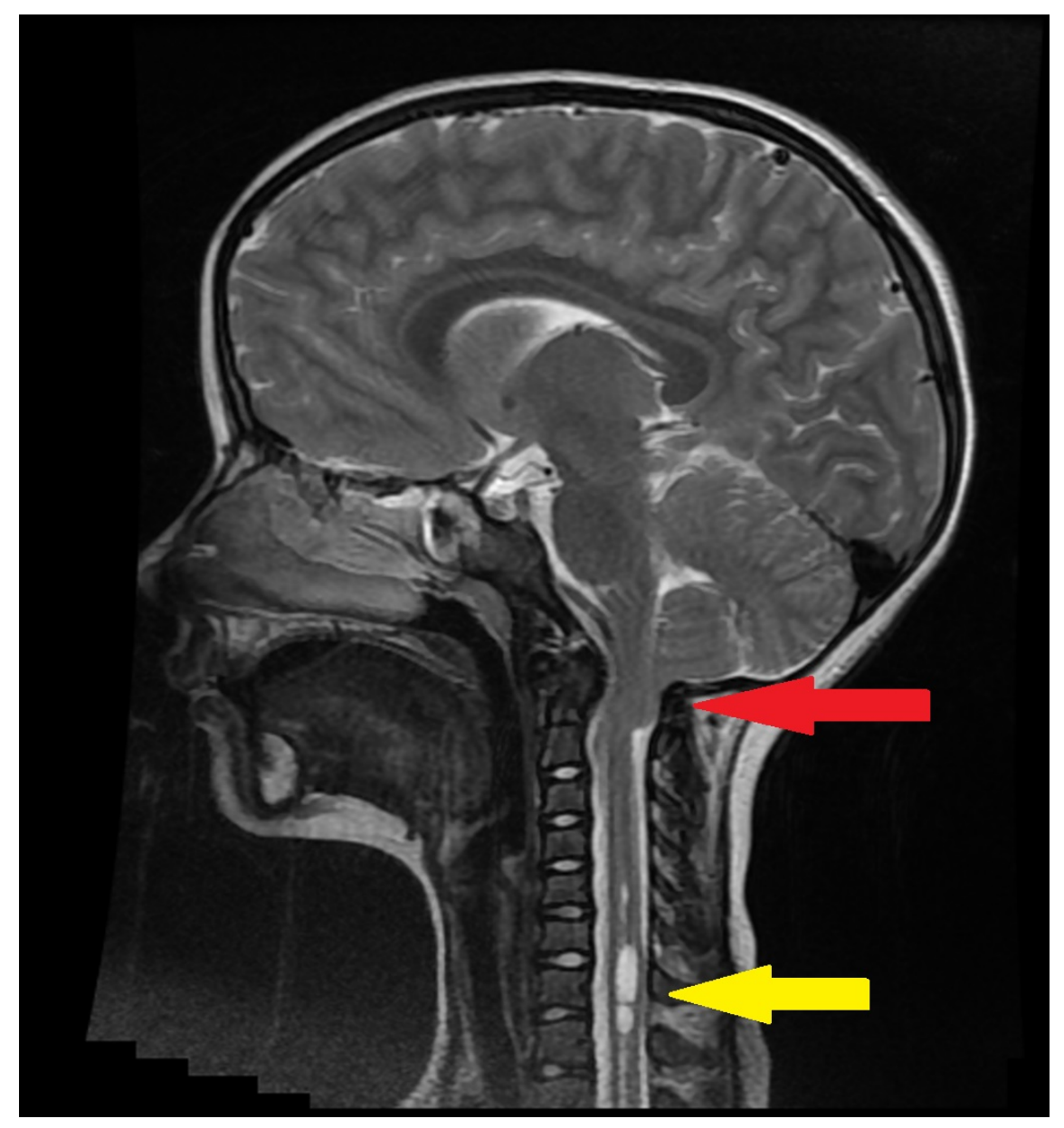

